\title{
Waveguiding without a waveguide: Local-mode exciton polaritons in multiple quantum wells
}

\author{
D. S. Citrin a) \\ Center for Ultrafast Optical Science, The University of Michigan, Ann Arbor, Michigan 48109-2099
}

(Received 25 May 1994; accepted for publication 9 December 1994)

\begin{abstract}
Exciton polaritons are considered in infinite multiple quantum wells which are periodic except that one well (impurity) has a different well width from the others (host). Under certain circumstances it is possible to have a long-lived exciton-polariton mode localized about the impurity. From the viewpoint of the electromagnetic field, this corresponds to waveguide modes in the vicinity of an exciton resonance in such structures. (C) 1995 American Institute of Physics.
\end{abstract}

Exciton polaritons in a single semiconductor quantum well (QW) are unsuitable to be the basis of a waveguide for the following reason. Consider first a defect-free, infinite, periodic multiple QW (MQW). Because of the translational invariance in all directions, an exciton in such a structure is unable to undergo radiative decay. Instead, the exciton and the electromagnetic field form coupled stationary states are known as exciton polaritons. ${ }^{1}$ Agranovich and Dubovskii, however, first noted that in low-dimensional systems one expects nonvanishing excitonic radiative widths for states with a sufficiently small wave vector, due to the relaxation of momentum conservation in directions normal to heterointerfaces. ${ }^{2}$ Thus, an exciton in a single QW or wire is too short lived to be useful for wave propagation. In this study we consider a structure of type intermediate between bulk and low-dimensional, namely an infinite MQW which is periodic in the growth $(\hat{\mathbf{z}})$ direction except that a single QW (impurity) in the stack differs from the other QW's (host). We find that if the exciton resonance associated with the impurity lies above that of the host, then for sufficiently small in-plane excitation wave vector $\mathbf{k}_{\|}$there is a long-lived exciton-polariton mode localized about the impurity. For the lower-branch nonradiative surface polaritons, the existence of a local mode depends on whether the impurity resonance lies above or below that of the host and on the direction of the dipole moment. The local exciton polaritons correspond to guided optical modes. Information on the polaritons is therefore of direct relevance to answering the question of the existence of waveguide modes in the vicinity of an exciton resonance. Provided the off-diagonal perturbation to the interwell radiative coupling can be neglected, the model is exactly solvable.

The polariton formalism is appropriate to describe the self-consistent coupled exciton-electromagnetic modes of the system. Because we are interested in resonant phenomena, we are justified in neglecting the small dielectric mismatch between well and barrier materials. ${ }^{3}$ We assume a spatially uniform background dielectric constant $\epsilon_{b}$ so that the speed of light in the medium in the absence of the exciton resonance is $c=c_{0} / \sqrt{\epsilon_{b}}$ with $c_{0}$ the value in vacuo. We consider a stack of $N=2 M+1$ symmetric QWs $(M \rightarrow \infty)$, label the QWs by the index $l$, and let $l=0$ be the impurity and

${ }^{a}$ Electronic mail: citrin@mail.physics.lsa.umich.edu
$l=-M, \ldots, M(l \neq 0)$ the host. The center-to-center spacing between successive QWs is $L$ and $L_{l}=l L$. We assume the absence of interwell electronic coupling which in practice often requires $L \gg \bar{L}_{z}, L_{z}$. The exciton (resonant) energy in QW $l$ is $E_{l}=E_{l}\left(\mathbf{k}_{\|}\right)$. It is assumed that $L_{z}$ and $\bar{L}_{z}$ are much less than the optical wavelength of the dipole transition of interest. We calculate the dipole $G F \stackrel{\leftrightarrow}{\mathrm{D}}$, defined in Ref. 4 . One shows that the polaritons can be described as $s$-wave (TE) and $p$-wave (TM). ${ }^{5}$ The $s$-mode corresponds to the $T$ polariton where the dipole moment lies in the QW plane but is perpendicular to $\mathbf{k}_{\|}$while the $p$-modes (in a single symmetric QW) can be further classified as the $L$-mode (dipole moment parallel to $\mathbf{k}_{\|}$) and the $Z$-mode (dipole moment parallel to $\mathbf{z}$ direction). We assume that there is no electronic coupling between the $T, L$, and $Z$ modes, which holds exactly for the heavy- and light-hole excitons in zincblende semiconductor QWs. In MQWs, the $L$ and $Z$ modes are coupled optically; the magnitude of the LT-Z splitting, however, implies that this coupling is weak in practice for GaAs/AlGaAs $\mathrm{MQWs}^{4}$ and is therefore neglected here, allowing us to drop the dyadic notation and write $D_{\epsilon}$ with $\epsilon \in\{T, L, Z\}$.

The GF satisfies the Dyson equation

$$
\mathrm{D}^{-1}=\mathrm{D}^{(0)-1}-\hbar \Sigma^{(1)}-\hbar \Sigma^{(2)},
$$

where $\mathrm{D}=\mathrm{D}_{\epsilon}(k)$ and $\left[\mathrm{D}^{(0)}\right]_{l l^{\prime}}=D_{l}^{(0)} \delta_{l l^{\prime}}$ with $D_{l}^{(0)}$ $=2 E_{l} /\left[(i \epsilon)^{2}-E_{l}^{2}\right]$ is the exciton GF in the absence of the coupling to the electromagnetic field and $i \epsilon$ is the energy variable (complex). We employ the abbreviation $k$ $=\left(i \epsilon, \mathbf{k}_{\|}\right) \cdot \hbar \Sigma^{(1)}=\hbar \Sigma_{\epsilon}^{(1)}(k)$ is the spatially regular part of the radiative self-energy (SE). ${ }^{4}$ Its matrix elements in the site basis are $\hbar \Sigma_{\epsilon l l^{\prime}}^{(1)}=F_{l l^{\prime}}\left[\hbar \Sigma_{\epsilon l^{\prime}}^{(1)}(k) \hbar \Sigma_{\epsilon l^{\prime}}^{(1)}(k)\right]^{1 / 2}$ with $\hbar \Sigma_{\epsilon l}^{(1)}(k)$ the single-QW SE associated with QW $l,{ }^{4}$ $F_{l l^{\prime}}=\exp \left(-\alpha\left|L_{l}-L_{l^{\prime}}\right|\right), \quad \alpha=\left[k_{\|}^{2}-(i \kappa)^{2}\right]^{1 / 2}, \quad$ and $i \kappa=i \epsilon /(\hbar c)$. $\hbar \Sigma^{(1)}$ accounts for the radiative coupling both within a single QW and between different QWs. One shows ${ }^{4} \hbar \Sigma_{\epsilon l}^{(1)}\left(\mathbf{k}_{\|}, E+i 0^{+}\right)$ $=\hbar \Pi_{\epsilon l}\left(\mathbf{k}_{\|}, E\right) \theta\left(k_{\|}-\kappa\right)-i \hbar \Gamma_{\epsilon l}\left(\mathbf{k}_{\|}, E\right) \theta\left(\kappa-k_{\|}\right) \quad$ where $\hbar \Gamma_{\epsilon l}>0$ and $\hbar \Pi_{T, Z l}<0$ and $\hbar \Pi_{L l}>0[\kappa=(E$ $\left.\left.+i 0^{+}\right) /(\hbar c)\right]$. For $k_{\|}<\kappa, \hbar \Gamma_{\epsilon l}$ gives a nonvanishing radiative width to the corresponding mode in a single QW. For $k_{\|}>\kappa$, the modes are purely stationary (surface polariton). $\hbar \Sigma^{(2)}=\hbar \Sigma_{\epsilon}^{(2)}(k)$ where $\hbar \Sigma_{\epsilon l l^{\prime}}^{(2)} ;(k)=\hbar \Sigma_{\epsilon l}^{(2)}\left(\mathbf{k}_{\|}\right) \delta_{l l^{\prime}}$ is the spatially singular SE which gives rise to the $L T-Z$ splitting. ${ }^{5}$ This term is nonzero only for $\epsilon=Z$ and is real and energy 
independent. Thus, it only enters the theory as a renormalization of $E_{l}{ }^{4}{ }^{4}$ The reader will find explicit expressions for all these quantities in Ref. 4 . In the sequel the $\epsilon, \mathbf{k}_{\|}$dependence, where omitted, is to be understood.

Equation (1) is exact in the polariton model regardless of the interwell spacings, resonant energies, and oscillator strengths of the various QWs. We write $E_{l}=\bar{E}+\Delta_{l}$, $\hbar \mathbf{\Sigma}^{(1)}=\hbar \overline{\boldsymbol{\Sigma}}^{(1)}+\boldsymbol{\Delta}^{(1)}+\boldsymbol{\Delta}^{\prime(1)}$, and $\hbar \boldsymbol{\Sigma}^{(2)}=\hbar \bar{\Sigma}^{(2)} 1+\boldsymbol{\Delta}^{(2)}$ with $\Delta_{0}=\Delta$ and $l=0$, and $\Delta_{l}=0$ otherwise. $\hbar \overline{\mathbf{\Sigma}}^{(1)}$ and $\hbar \overline{\mathbf{\Sigma}}^{(2)}$ are the SE's of the host. Since $\hbar \mathbf{\Sigma}^{(2)}$ is site diagonal, so is $\boldsymbol{\Delta}^{(2)} \cdot \boldsymbol{\Delta}^{(1)}$ is defined to be the site-diagonal part of $\hbar \mathbf{\Sigma}^{(1)}-\hbar \overline{\boldsymbol{\Sigma}}^{(1)}$ and $\boldsymbol{\Delta}^{\prime(1)}$ is the off-diagonal part with $\Delta_{l l}^{\prime(1)}=0$. Quantities with bars are characteristic of the host. The inverse GF is split as

$$
\mathrm{D}^{-1}=\overline{\mathrm{D}}^{-1}-\sigma-\Delta^{\prime-1},
$$

with

$$
\begin{aligned}
& \overline{\mathrm{D}}^{-1}=\overline{\mathrm{D}}^{(0)-1}-\hbar \overline{\mathbf{\Sigma}}^{(1)}-\hbar \bar{\Sigma}^{(2)} 1, \\
& \overline{\mathrm{D}}^{(0)}=2 \bar{E} 1 /\left[(i \epsilon)^{2}-\bar{E}^{2}\right], \\
& \boldsymbol{\sigma}=\boldsymbol{\Xi}+\boldsymbol{\Delta}^{(1)}+\boldsymbol{\Delta}^{(2)}, \\
& \Delta_{l l}^{(1)}=\Delta^{(1)} \delta_{l 0} \delta_{l^{\prime} 0,}
\end{aligned}
$$

and

$$
\Delta_{l l}^{\prime(2)}=\Delta^{(2)} \delta_{l 0} \delta_{l^{\prime} 0}
$$

These equations define $\Xi$.

We pause to consider the various terms in Eq. (2). The $G F \overline{\mathrm{D}}$ describes the exciton polariton in the host MQW with $\boldsymbol{\sigma}=\boldsymbol{\Delta}^{\prime(1)}=0$. The impurity effect is contained in $\boldsymbol{\sigma}$ $+\boldsymbol{\Delta}^{\prime(1)}$. The term $\boldsymbol{\sigma}$ is the site-diagonal part of the impurity term, which in turn, is composed of a contribution $\Xi$ due to the difference in the resonant energy of the impurity while $\Delta^{(1)}$ and $\Delta^{(2)}$ account for the difference in the oscillator strength of the impurity. For $k_{\|}<\kappa, \Delta^{(1)} \in i \Re$. For such cases $\boldsymbol{\Delta}^{(1)}$ is responsible for radiative decay. $\boldsymbol{\Delta}^{(2)}$ accounts for differences in the $L T-Z$ splitting between the impurity and host. Since $\Xi\left(\bar{E}, \mathbf{k}_{\|}\right)=\boldsymbol{\Delta}$, for resonant phenomena the replacement $\boldsymbol{\Xi} \rightarrow \boldsymbol{\Delta}$ is justified. The off-diagonal impurity effect $\boldsymbol{\Delta}^{\prime(1)}$ accounts for the difference in the interwell radiative couplings between the impurity and host. Note that by utilizing a material for the impurity different from the host QWs one can attain independent control over $\Delta$ and $\Delta^{(1)}$. The present theory also provides a description of exciton polaritons in molecular heterostructures possessing an impurity layer.

We briefly review the properties of exciton polaritons in the host. For an infinite periodic MQW without an impurity, $\bar{D}$ is diagonal in the representation $\{|q\rangle\}$ where $\langle z \mid q\rangle=N^{-1 / 2} \exp (i q z)$, i.e., $[\overline{\mathrm{D}}]_{q q^{\prime}}=\bar{D}_{q} \delta_{q q^{\prime}}$. In this case, $\boldsymbol{\sigma}=\boldsymbol{\Delta}^{\prime(1)}=0 \quad$ and $\quad \bar{D}_{q}^{-1}=\bar{D}^{(0)-1}-\hbar \bar{\Sigma}^{(1)} F_{q}-\hbar \bar{\Sigma}^{(2)}$ with. ${ }^{4} \quad F_{q}=\sinh y /(\cosh y-\cos q L), \quad$ and $\quad y=\alpha L .{ }^{4}$ $\hbar \bar{\Sigma}^{(1)}$ is the SE associated with a single QW characteristic of the host. The poles of $\bar{D}_{q}$ are real which indicates the absence of excitonic radiative decay in the infinite MQW in which all QWs are equivalent. ${ }^{4}$

We now investigate the existence and stability of waveguide modes of the MQW associated with the impurity. This is accomplished by examining the resonances of a local GF defined at the impurity site, $l=0$. In general, the resonances possess an imaginary part of which there are two primary sources: scattering out of the state (nonexistence of a welldefined local mode) and radiative decay. We shall neglect the off-diagonal part $\boldsymbol{\Delta}^{\prime(1)}$ of the perturbation to the radiative coupling between QWs. The effect of this is commented upon later. We have $\Xi_{l l^{\prime}}=\Xi_{l}(k) \delta_{l l^{\prime}}$, with $\Xi_{l}(k)$ given by Eq. (2). It will simplify the ensuing discussion if we only retain the term in $\Xi_{l}$ to first order in $\Delta_{l}$, namely $\Xi_{l}(k)$ $\approx \Delta_{l}\left[1+\bar{E}^{-1} \bar{D}^{(0)-1}(k)\right] \approx \Delta_{l}$. No significant accuracy will be lost in the resonant region provided $\Delta \ll \bar{E}$. We can write the total GF as $\mathrm{D}=\overline{\mathrm{D}}+\overline{\mathrm{D}} \mathrm{TD}$ where the $t$-matrix is given by ${ }^{6} \quad T_{l l^{\prime}}=T \delta_{l 0} \delta_{l^{\prime} 0}$ and $T=\sigma /\left(1-\sigma P_{0}\right)$ with $\sigma=\sigma_{00}$ and $\bar{D}_{l l^{\prime}}=P_{l-l^{\prime}}=(L / 2 \pi) \int_{0}^{2 \pi / L} d q$ $\times \exp \left[\left(l-l^{\prime}\right) q L\right] \bar{D}_{q}$ :

$$
\begin{aligned}
& P_{l}= \begin{cases}\chi\left(1-\zeta_{0}\right) & l=0 \\
-\chi \zeta_{|l|} & |l| \geqslant 1\end{cases} \\
& \zeta_{|l|}=\zeta_{0}\left\{\left[\left(1-\tilde{a}^{2}\right)^{1 / 2}-1\right] / \tilde{a}\right\}^{|l|},
\end{aligned}
$$

with

$$
\begin{aligned}
& \zeta_{0}=\left(1-\tilde{a}^{2}\right)^{-1 / 2}(\tilde{a} \cosh y+1), \\
& \chi=(2 \bar{E}) /\left[(i \epsilon)^{2}-\bar{E}^{2}-2 \bar{E} \hbar \bar{\Sigma}^{(2)}\right],
\end{aligned}
$$

and

$$
\tilde{a}^{-1}=\left(\chi \hbar \bar{\Sigma}^{(1)} \tanh y-1\right) \cosh y .
$$

Note that $P_{0}=N^{-1} \operatorname{Tr} \overline{\mathrm{D}}$. Thus, for the impurity-free MQW, the density of states at fixed $\epsilon$ and $\mathbf{k} \|$ is proportional to $-\operatorname{Im} P_{0}\left(E+i 0^{+}\right)$. Moreover, $P_{0}\left(E+i 0^{+}\right)$is proportional to the transmission coefficient $t(E)$ for electromagnetic waves through the MQW stack (when we properly account for the $L-Z$ coupling): $t(E)=(-2 \pi / \alpha)$ $\times P_{0}\left(E+i 0^{+}\right){ }^{4}$

The local GF at the impurity location is $D_{00}$ $=P_{0} /\left(1-\sigma P_{0}\right)$ whose poles give the local modes. Thus $D_{00}^{-1}=0$ can be regarded as the dispersion relation for the waveguide mode, $\chi^{-1}=\sigma\left(1-\zeta_{0}\right)$. Equation (3) gives a long-lived local mode if $|\tilde{a}|<1$ or approximately

$$
\left|\hbar \bar{\Sigma}^{(1)} \Delta^{-1} \tanh y-1\right||\cosh y|>1 .
$$

We expect the local mode to be at $\sim \bar{E}+\Delta$. The dispersion relation can be written

$$
E^{2}-\bar{E}^{2}-\hbar \Sigma_{\epsilon, l=0}^{(2)} \approx \Delta\left(1-\zeta_{0}\right)+\Delta^{(1)}
$$

All energy arguments in Eq. (5) are evaluated withi $\epsilon \rightarrow \bar{E}$ $+i 0^{+}$. The local mode, provided it exists, is radiatively unstable with a lifetime given by $-\hbar / \operatorname{Im} \Delta^{(1)}$. For the GaAs/ AlGaAs system with $\bar{L}_{z}, L_{z}<15 \mathrm{~nm},\left|\Delta^{(1)}\right|$ is of the order of $0.01-0.1 \mathrm{meV}$. If $k_{\|}<\kappa$, then $\Delta^{(1)}=\hbar \Sigma_{l=0}^{(1)}$ $-\hbar \bar{\Sigma}^{(1)} \in i \Re$. For GaAs/AlGaAs-based structures, the typical decay radiative rates associated with $\hbar \Sigma_{l=0}^{(1)}$ and $\hbar \bar{\Sigma}^{(1)}$ are one of the order of $10 \mathrm{ps} .{ }^{5,7}$ We therefore, expect typical values of $\left|\hbar / \Delta^{(1)}\right|$ to be greater than 100 ps. This implies polariton propagation over distances on the order of at least $1 \mathrm{~mm}$ before the exciton polariton has undergone radiative decay. This discussion neglects the $\mathbf{k}_{\|}$dependence, dephasing, scattering off static disorder, and finite-size ef- 
fects in real MQW structures. Nevertheless, the foregoing indicates that for many cases of interest we can neglect $\Delta^{(1)}$. Thus, the local mode experiences strongly inhibited radiative decay compared with the exciton polariton in a single QW without the host present. ${ }^{4,5}$ We note that if the oscillator strength of the impurity is lower than that of the host, the sign of the imaginary part of $\Delta^{(1)}$ is positive which implies that the state is unstable in time with respect to growth. This indicates a tendency for the exciton polariton to self-trap at the impurity site. The retarded temporal boundary conditions dictate that the modes cannot in fact be exponentially growing; therefore, the off-diagonal term $\boldsymbol{\Delta}^{\prime(1)}$ must eventually stabilize the time dependence.

Equation (4) gives the condition for the existence of a local mode. It will prove useful to have estimates of the parameters of relevance to one of the most commonly employed material system from which semiconductor heterostructures are fabricated, namely GaAs/AlGaAs. In this case $\kappa^{-1} \sim 40 \mathrm{~nm}$. For structures with $\bar{L}_{z}, L_{z}<15 \mathrm{~nm}$, monolayer differences between $\bar{L}_{z}$ and $L_{z}$ result in $\Delta$ on the order of or greater than $1 \mathrm{meV}$. Thus, $\left|\hbar \bar{\Sigma}^{(1)}\left(0, E+i 0^{+}\right) / \Delta\right|$ is typically less than 0.1 . We define the long-wavelength limit (LWL) by $\kappa L \ll 1$. Thus, for GaAs/AlGaAs structures, the LWL requires $L \ll 40 \mathrm{~nm}$. In the LWL, Eq. (4) gives $\left|\hbar \bar{\Sigma}^{(1)} y / \Delta-1\right|>1$ as the condition for the existence of a local mode. For $k_{\|}<\kappa$ (radiative modes for a single QW), $y \rightarrow-i L \sqrt{\kappa^{2}-k_{\|}^{2}}$ and we find a local mode exists if $\Delta>0$. Note that this mode lies within the exciton band of the host. We point out that the local mode is a stable collective excitation of the crystal. In practice, exciton-exciton and other scattering will couple the local state to the continuum states of the host leading to a Fano resonance and some degree of incoherent processes which gives rise to absorption of the local mode by the host continuum modes. For $k_{\|}>\kappa$, the sign of $\hbar \Pi_{\epsilon, l=0}$ gives for $\Delta>0$ the $T$ and $Z$ polarizations support local modes while for $\Delta<0$ the $L$ polarization supports a local mode.

The $l$ dependence of the normalized local-mode amplitude is $D_{0 l} / D_{00}=P_{l}$ evaluated at the local-mode resonance. If the local mode exists, then Eq. (3) gives that the amplitude falls off exponentially as $\exp \left(-|l| \ln \left\{\tilde{a} /\left[\left(1-\tilde{a}^{2}\right)^{1 / 2}\right.\right.\right.$ $-1]\})$. In the LWA, this is $\exp \{-|l|$ $\left.\times\left[-\Delta /\left(2 \hbar \bar{\Sigma}^{(1)} y\right)\right]^{1 / 2}\right\}$. Thus, the localization length falls off with decreasing coupling strength $\left|\hbar \bar{\Sigma}^{(1)}\right|$ and interwell spacing $L$ and with increasing impurity potential $|\Delta|$ and wavelength $2 \pi \kappa^{-1}$. Consider a GaAs/AlGaAs MQW with $\bar{L}_{z}=10, \quad L_{z}=9.5$, and $L=20 \mathrm{~nm}$. Then $\left|\hbar \bar{\Sigma}^{(1)} / \Delta\right|$ $\approx 0.05$, which implies that the excitation is localized over a region which includes approximately five QWs on each side of the impurity.

In this study, we have demonstrated the existence of long-lived local exciton-polariton modes in a MQW in which one QW differs from the others. These local exciton polaritons can be viewed as optical waveguide modes in the vicinity of the exciton resonance. It is noteworthy that by way of contrast, in the absence of the host, the radiative lifetime of the exciton in the impurity QW is very short- $\sim 10 \mathrm{ps}$ in GaAs/AlGaAs QWs. Thus, the presence of the host inhibits spontaneous emission, and this inhibition is achieved without employing an optical cavity. The model presented, however, has neglected other excitonic resonances. For example, if $\Delta>0$ and there is a higher-lying host resonance near $\bar{E}$ $+\Delta$, then the local mode as calculated above will not be stable.

One can envisage a number of novel applications based on the proposed waveguide structure. Because the waveguiding relies on the presence of an active medium, the existence, and nature of the guided modes will depend upon the state of the various QWs. For example, by saturating the host transition $\bar{E}$, the host oscillators will effectively be removed from participating in the formation of collective modes thus reallowing rapid radiative recombination of the local-mode exciton polariton. Although the present calculation deals with a single impurity $\mathrm{QW}$ in a periodic host, one expects other configurations also to exhibit similar local modes. For example, if $L$ is not too small, one can make use of an impurity layer composed of two closely spaced QWs, thereby effectively doubling the oscillator strength of the impurity transition, while leaving its resonant energy unchanged. In order that the effects discussed be robust, optically thick samples are required. Otherwise, the waveguide modes will be too leaky to make effective use of them.

This work was supported by the National Science Foundation through the Fellows Program of the Center for Ultrafast Optical Science under STC PHY 8920108.

${ }^{1}$ J. J. Hopfield, Phys. Rev. 112, 1555 (1958).

${ }^{2}$ V. M. Agranovich and A. O. Dubovskii, JETP Lett. 3, 223 (1966).

${ }^{3}$ S. Jorda, J. Phys. Paris C5-3, 59 (1993).

${ }^{4}$ D. S. Citrin, Phys. Rev. B 47, 3832 (1993); Solid State Commun. 89, 139 (1994); Phys. Rev. B 50, 5497 (1994).

${ }^{5}$ L. C. Andreani and F. Bassani, Phys. Rev. B 41, 7536 (1990); L. C. Andreani, F. Tassone, and F. Bassani, Solid State Commun. 77, 641 (1991).

${ }^{6}$ R. J. Elliott, J. A. Krumhansl, and P. L. Leath, Rev. Mod. Phys. 46, 465 (1974).

${ }^{7}$ B. Deveaud, F. Clérot, N. Roy, K. Satzke, B. Sermage, and D. S. Katzer, Phys. Rev. Lett. 67, 2355 (1991). 